\title{
Combined Convex Technique on Delay-Distribution-Dependent Stability for Delayed Neural Networks
}

\author{
Ting Wang, Tao Li, Mingxiang Xue, and Shumin Fei \\ Key Laboratory of Measurement and Control of CSE, School of Automation, Southeast University, \\ Ministry of Education, Nanjing 210096, China \\ Correspondence should be addressed to Ting Wang, chunchun@163.com
}

Received 26 March 2012; Revised 27 May 2012; Accepted 27 May 2012

Academic Editor: Zhengqiu Zhang

Copyright (C 2012 Ting Wang et al. This is an open access article distributed under the Creative Commons Attribution License, which permits unrestricted use, distribution, and reproduction in any medium, provided the original work is properly cited.

\begin{abstract}
Together with the Lyapunov-Krasovskii functional approach and an improved delay-partitioning idea, one novel sufficient condition is derived to guarantee a class of delayed neural networks to be asymptotically stable in the mean-square sense, in which the probabilistic variable delay and both of delay variation limits can be measured. Through combining the reciprocal convex technique and convex technique one, the criterion is presented via LMIs and its solvability heavily depends on the sizes of both time-delay range and its variations, which can become much less conservative than those present ones by thinning the delay intervals. Finally, it can be demonstrated by four numerical examples that our idea reduces the conservatism more effectively than some earlier reported ones.
\end{abstract}

\section{Introduction}

In past decades, neural networks have been applied to various signal processing problems, such as optimization, image processing, associative memory design, and other engineering fields. In those applications, the key feature of the designed neural network is to be globally stable. Meanwhile, since there inevitably exists communication delay which can induce the oscillation and instability in various dynamical systems, great efforts have been made to analyze the dynamics of delayed systems including delayed neural networks (DNNs) and many elegant results have been reported; see [1-35]. In practical applications, though it is difficult to describe the form of time-delay precisely, the bounds of timedelay and its variation rates still can be measured. Since the Lyapunov functional approach imposes no restriction on delay variation and presents some simple stability criteria, the Lyapunov-Krasovskii functional (LKF) has been widely utilized due to that its analysis can 
fully make use of the information on time-delay of DNNs as much as possible. Thus recently, the delay-dependent stability has become an important topic of primary significance, in which the main purpose is to derive an allowable delay upper bound guaranteeing the global stability for addressed DNNs in [4-9, 16, 17, 21-35]. Furthermore, in recent years, since the delay-partitioning ideas have been proven to be more effective in reducing the conservatism than some previously reported techniques and received much research attention in [2127], yet these convex ideas above still need some further improvements since they cannot effectively tackle interval variable delay or cannot fully utilize every delay subintervals, which have been fully addressed in [28].

Meanwhile, it can be seen from many existing references that only the deterministic time-delay case was concerned, and the stability criteria were derived based on the information of delay and its variation range. Actually, time-delay in some DNNs is often existent in a stochastic fashion. In practice, to propagate and control the stochastic signals through universal learning networks, a probabilistic universal leaning network (PULN) was proposed. In a PULN, the output signal of the node is transferred to another node by multibranches with arbitrary time-delays which are random and its probability often can be measured by the statistical methods. For this case, if some values of time-delay are very large but its probability of the delay taking such large values is very small, it may lead to a more conservative result if only the information of delay variation range is considered. Thus, recently, some researchers have considered the stability for various systems including DNNs with probability-distributed delays [29-39]. In [36-39], the authors have analyzed the stability and its applications for networked control systems, uncertain linear systems, and T-S fuzzy systems, in which probability delay has been fully considered. As for discretetime DNNs with probabilistic delay, the global stability has been considered and some pretty results have been proposed in [29-33]. Yet, it has come to our attention that, though some works have studied the dynamics of continuous-time DNNs with probabilistic delay [34, 35], the lower limits of delay variation have not been considered and, in fact, such available information could play an important role in extending the results' application area, which has been illustrated in [40] and yet not taking delay distribution probability into consideration. Presently, as for time-variable delay, the reciprocal convex approach in [41] has been proven to be more effective in reducing the conservatism than some earlier convex techniques $[28,42]$. Yet to the authors' best knowledge, few authors have used the combination of the reciprocal convex technique and general convex ones to tackle the global stability for DNNs with probabilistic time-varying delay, which constitutes the main focus of this present work.

Together with taking both bounds on probabilistic time-delay and its time variations into consideration, we make some great efforts to investigate the mean-squared stability for DNNs, in which an improved delay-partitioning idea is utilized and a novel Lyapunov functional is chosen. Through combining the reciprocal convex technique and the general convex one, one less conservative condition is given in terms of LMIs, which can present the pretty delay dependence and computational efficiency. Finally, we give four numerical examples to illustrate that our derived results can be less conservative than some existent ones.

The notations in the paper are really standard. For symmetric matrices $X, Y, X>Y$ (resp., $X \geq Y)$ means that $X-Y>0(X-Y \geq 0)$ is a positive-definite (resp., positivesemidefinite) matrix; and $*$ denotes the symmetric term in a symmetric matrix, that is, $\left[\begin{array}{cc}X & Y \\ Y^{T} & Z\end{array}\right]=\left[\begin{array}{ll}X & Y \\ * & Z\end{array}\right]$. 


\section{Problem Formulations and Preliminaries}

Consider the delayed neural networks as follows:

$$
\dot{z}(t)=-C z(t)+A g(z(t))+B g(z(t-\tau(t)))+\mathrm{I},
$$

where $z=\left[z_{1}, \ldots, z_{n}\right]^{T} \in \mathbf{R}^{n}$ is a real $n$-vector denoting the state variables associated with the neurons, $g(z)=\left[g_{1}\left(z_{1}\right), \ldots, g_{n}\left(z_{n}\right)\right]^{T}$ represents the neuron activation function, $\mathrm{I}=\left[\mathrm{I}_{1}, \ldots, \mathrm{I}_{n}\right]^{T} \in \mathbf{R}^{n}$ is a constant input vector, $C=\operatorname{diag}\left\{c_{1}, \ldots, c_{n}\right\}>0$, and $A, B$ are the appropriately dimensional constant matrices.

The following assumptions on the system (2.1) are made throughout this paper.

Assumption 2.1. The time-varying delay $\tau(t)$ satisfies $0 \leq \tau_{1} \leq \tau(t) \leq \tau_{3}$. Moreover, consider the information of probability distribution of $\tau(t)$, two sets and functions are defined as $\Omega_{1}=$ $\left\{t, \tau(t) \in\left[\tau_{1}, \tau_{2}\right)\right\}, \Omega_{2}=\left\{t, \tau(t) \in\left[\tau_{2}, \tau_{3}\right]\right\}$, and

$$
\begin{gathered}
\tau_{1}(t)= \begin{cases}\tau(t), & \text { for } t \in \Omega_{1}, \\
\bar{\tau}_{1}, & \text { for } t \in \Omega_{2},\end{cases} \\
\tau_{2}(t)= \begin{cases}\tau(t), & \text { for } t \in \Omega_{2}, \\
\bar{\tau}_{2}, & \text { for } t \in \Omega_{1},\end{cases} \\
\mu_{1} \leq \dot{\tau}_{1}(t) \leq \mu_{2}, \quad \mu_{3} \leq \dot{\tau}_{2}(t) \leq \mu_{4},
\end{gathered}
$$

where $\tau_{2} \in\left[\tau_{1}, \tau_{3}\right], \bar{\tau}_{1} \in\left[\tau_{1}, \tau_{2}\right)$, and $\bar{\tau}_{2} \in\left[\tau_{2}, \tau_{3}\right]$. It is easy to check that $t \in \Omega_{1}$ means that the event $\tau(t) \in\left[\tau_{1}, \tau_{2}\right)$ occurs and $t \in \Omega_{2}$ means that the event $\tau(t) \in\left[\tau_{2}, \tau_{3}\right]$ occurs. Therefore, a stochastic variable $\delta(t)$ can be defined as

$$
\delta(t)= \begin{cases}1, & \text { for } t \in \Omega_{1} \\ 0, & \text { for } t \in \Omega_{2}\end{cases}
$$

Assumption 2.2. $\delta(t)$ is a Bernoulli distributed sequence with

$$
\begin{aligned}
& \operatorname{Prob}\{\delta(t)=1\}=\mathbf{E}\{\delta(\mathbf{t})\}=\delta_{0}, \\
& \operatorname{Prob}\{\delta(t)=0\}=1-\mathbf{E}\{\delta(\mathbf{t})\}=1-\delta_{0},
\end{aligned}
$$

where $0 \leq \delta_{0} \leq 1$ is a constant and $\mathbf{E}\{\delta(\mathbf{t})\}$ is the mathematical expectation of $\delta(t)$. It is easy to check that $\mathbf{E}\left\{\delta(\mathbf{t})-\delta_{0}\right\}=0$.

Assumption 2.3. For the constants $\rho_{j}^{+}, \rho_{j}^{-}$, the nonlinear function $g_{j}(\cdot)$ in (2.1) satisfies the following condition:

$$
\rho_{j}^{-} \leq \frac{g_{j}(\alpha)-g_{j}(\beta)}{\alpha-\beta} \leq \rho_{j}^{+}, \quad \forall \alpha, \beta \in \mathbf{R}, \alpha \neq \beta, j=1,2, \ldots, n
$$


Here, we denote $\bar{\Sigma}=\operatorname{diag}\left\{\rho_{1}^{+}, \ldots, \rho_{n}^{+}\right\}, \Sigma=\operatorname{diag}\left\{\rho_{1}^{-}, \ldots, \rho_{n}^{-}\right\}$, and

$$
\Sigma_{1}=\operatorname{diag}\left\{\rho_{1}^{+} \rho_{1}^{-}, \ldots, \rho_{n}^{+} \rho_{n}^{-}\right\}, \quad \Sigma_{2}=\operatorname{diag}\left\{\frac{\rho_{1}^{+}+\rho_{1}^{-}}{2}, \ldots, \frac{\rho_{n}^{+}+\rho_{n}^{-}}{2}\right\}
$$

It is clear that under Assumptions 2.1-2.3, system (2.1) has one equilibrium point $z^{*}=$ $\left[z_{1}^{*}, \ldots, z_{n}^{*}\right]^{T}$. For convenience, we shift the equilibrium point $z^{*}$ to the origin by letting $x=z-z^{*}, f(x)=g\left(x+z^{*}\right)-g\left(z^{*}\right)$, and the system (2.1) can be converted to

$$
\dot{x}(t)=-C x(t)+A f(x(t))+B f(x(t-\tau(t))),
$$

where $f(x)=\left[f_{1}\left(x_{1}\right), \ldots, f_{n}\left(x_{n}\right)\right]^{T}$. Based on the methods in [37-39], the system above can be equivalently converted to

$$
\dot{x}(t)=-C x(t)+A f(x(t))+\delta(t) B f\left(x\left(t-\tau_{1}(t)\right)\right)+(1-\delta(t)) B f\left(x\left(t-\tau_{2}(t)\right)\right) .
$$

It is easy to check that the function $f_{j}(\cdot)$ satisfies $f_{j}(0)=0$, and

$$
\rho_{j}^{-} \leq \frac{f_{j}(\alpha)}{\alpha} \leq \rho_{j}^{+}, \quad \forall \alpha \in \mathbf{R}, \alpha \neq 0, j=1,2, \ldots, n .
$$

Then, the problem to be addressed in the paper can be formulated as developing a condition ensuring that the system (2.9) is asymptotically stable. introduced.

In order to obtain the stability criterion for system (2.9), the following lemmas are

Lemma 2.4 (see [27]). For any constant matrix $X \in \mathbf{R}^{n \times n}, X=X^{T} \geq 0$, a scalar functional $h:=h(t) \geq 0$, and a vector function $\dot{x}:[-h, 0] \rightarrow \mathbf{R}^{n}$ such that the following integration is well defined, then $-h \int_{t-h}^{h} \dot{x}^{T}(s) X \dot{x}(s) d s \leq[x(t)-x(t-h)]^{T} X[x(t)-x(t-h)]$.

Lemma 2.5 (see [41]). Let the functions $f_{1}(t), f_{2}(t), \ldots, f_{N}(t): \mathbf{R}^{m} \rightarrow \mathbf{R}$ have the positive values in an open subset $\mathbf{D}$ of $\mathbf{R}^{m}$ and satisfy $\left(1 / \alpha_{1}\right) f_{1}(t)+\left(1 / \alpha_{2}\right) f_{2}(t)+\cdots+\left(1 / \alpha_{N}\right) f_{N}(t): \mathbf{D} \rightarrow \mathbf{R}$ with $\alpha_{i}>0$ and $\sum_{i=1}^{N} \alpha_{i}=1$, then the reciprocal convex technique of $f_{i}(t)$ over the set $\mathbf{D}$ satisfies

$$
\sum_{i} \frac{1}{\alpha_{i}} f_{i}(t) \geq \sum_{i} f_{i}(t)+\sum_{i \neq j} g_{i, j}(t), \quad \forall g_{i, j}(t): \mathbf{R}^{m} \longrightarrow \mathbf{R},\left[\begin{array}{cc}
f_{i}(t) & g_{i, j}(t) \\
g_{i, j}^{T}(t) & f_{j}(t)
\end{array}\right] \geq 0
$$

\section{Delay-Distribution-Dependent Stability}

Firstly, we can rewrite the system (2.9) as

$$
\begin{aligned}
\dot{x}(t)= & -C x(t)+A f(x(t))+\delta_{0} B f\left(x\left(t-\tau_{1}(t)\right)\right)+\left(1-\delta_{0}\right) B f\left(x\left(t-\tau_{2}(t)\right)\right)+\left(\delta(t)-\delta_{0}\right) \\
& \times B\left[f\left(x\left(t-\tau_{1}(t)\right)\right)-f\left(x\left(t-\tau_{2}(t)\right)\right)\right] .
\end{aligned}
$$


Now letting $l, m$ be positive integers, we, respectively, divide the delay intervals $\left[\tau_{1}, \tau_{2}\right]$ and $\left[\tau_{2}, \tau_{3}\right]$ into $l, m$ segments averagely. Moreover, we introduce the following denotations:

$$
\delta_{1}=\frac{\tau_{2}-\tau_{1}}{l}, \quad \delta_{2}=\frac{\tau_{3}-\tau_{2}}{m}, \quad \rho_{1}(t)=\frac{\tau_{1}(t)-\tau_{1}}{l}, \quad \rho_{2}(t)=\frac{\tau_{2}(t)-\tau_{2}}{m} .
$$

Then, based on (2.10) and (3.2), we can construct the following Lyapunov-Krasovskii functional candidate:

$$
V\left(x_{t}\right)=V_{1}\left(x_{t}\right)+V_{2}\left(x_{t}\right)+V_{3}\left(x_{t}\right)
$$

where

$$
\begin{aligned}
& V_{1}\left(x_{t}\right)=x^{T}(t) P x(t)+\int_{-\tau_{1}}^{0} \int_{t+\theta}^{t} \tau_{1} \dot{x}^{T}(s) Q \dot{x}(s) d s d \theta \\
& +2 \sum_{i=1}^{n} k_{i} \int_{0}^{x_{i}}\left[f_{i}(s)-\rho_{i}^{-} s\right] d s+2 \sum_{i=1}^{n} l_{i} \int_{0}^{x_{i}}\left[\rho_{i}^{+} s-f_{i}(s)\right] d s, \\
& V_{2}\left(x_{t}\right)=\int_{t-\tau_{1}}^{t}\left[\begin{array}{c}
x(s) \\
f(x(s))
\end{array}\right]^{T}\left[\begin{array}{cc}
P_{1} & H_{1} \\
* & Q_{1}
\end{array}\right]\left[\begin{array}{c}
x(s) \\
f(x(s))
\end{array}\right] d s \\
& +\sum_{i=1}^{2} \int_{t-\tau_{i}-\delta_{i}}^{t-\tau_{i}}\left[\begin{array}{c}
\sigma_{i}(s) \\
h_{i}\left(\sigma_{i}(s)\right)
\end{array}\right]^{T}\left[\begin{array}{cc}
\widetilde{P}_{i} & \widetilde{H}_{i} \\
* & \widetilde{Q}_{i}
\end{array}\right]\left[\begin{array}{c}
\sigma_{i}(s) \\
h_{i}\left(\sigma_{i}(s)\right)
\end{array}\right] d s \\
& +\sum_{i=1}^{l} \int_{t-\tau_{1}-(i-1) \delta_{1}-\rho_{1}(t)}^{t-\tau_{1}-(i-1) \delta_{1}}\left[\begin{array}{c}
x(s) \\
f(x(s))
\end{array}\right]^{T}\left[\begin{array}{cc}
X_{1 i} & Y_{1 i} \\
* & Z_{1 i}
\end{array}\right]\left[\begin{array}{c}
x(s) \\
f(x(s))
\end{array}\right] d s \\
& +\sum_{i=1}^{l} \int_{t-\tau_{1}-i \delta_{1}}^{t-\tau_{1}-(i-1) \delta_{1}-\rho_{1}(t)}\left[\begin{array}{c}
x(s) \\
f(x(s))
\end{array}\right]^{T}\left[\begin{array}{cc}
X_{2 i} & Y_{2 i} \\
* & Z_{2 i}
\end{array}\right]\left[\begin{array}{c}
x(s) \\
f(x(s))
\end{array}\right] d s \\
& +\sum_{i=1}^{m} \int_{t-\tau_{2}-(i-1) \delta_{2}-\rho_{2}(t)}^{t-\tau_{2}-(i-1) \delta_{2}}\left[\begin{array}{c}
x(s) \\
f(x(s))
\end{array}\right]^{T}\left[\begin{array}{cc}
X_{3 i} & Y_{3 i} \\
* & Z_{3 i}
\end{array}\right]\left[\begin{array}{c}
x(s) \\
f(x(s))
\end{array}\right] d s \\
& +\sum_{i=1}^{m} \int_{t-\tau_{2}-i \delta_{2}}^{t-\tau_{2}-(i-1) \delta_{2}-\rho_{2}(t)}\left[\begin{array}{c}
x(s) \\
f(x(s))
\end{array}\right]^{T}\left[\begin{array}{cc}
X_{4 i} & Y_{4 i} \\
* & Z_{4 i}
\end{array}\right]\left[\begin{array}{c}
x(s) \\
f(x(s))
\end{array}\right] d s, \\
& V_{3}\left(x_{t}\right)=\sum_{i=1}^{l} \int_{-\tau_{1}-i \delta_{1}}^{-\tau_{1}-(i-1) \delta_{1}} \int_{t+\theta}^{t} \delta_{1} \dot{x}^{T}(s) W_{1 i} \dot{x}(s) d s d \theta \\
& +\sum_{i=1}^{m} \int_{-\tau_{2}-i \delta_{2}}^{-\tau_{2}-(i-1) \delta_{2}} \int_{t+\theta}^{t} \delta_{2} \dot{x}^{T}(s) W_{2 i} \dot{x}(s) d s d \theta,
\end{aligned}
$$


with $K=\operatorname{diag}\left\{k_{1}, \ldots, k_{n}\right\}, L=\operatorname{diag}\left\{l_{1}, \ldots, l_{n}\right\}, n \times n$ constant matrices $P, Q, P_{1}, H_{1}, Q_{1}$, $X_{j i}, Y_{j i}, Z_{j i}(j=1,2,3,4), W_{j i}(j=1,2), \ln \times \ln$ constant matrices $\widetilde{P}_{1}, \widetilde{Q}_{1}, \widetilde{H}_{1}, m n \times m n$ constant matrices $\widetilde{P}_{2}, \widetilde{Q}_{2}, \widetilde{H}_{2}$, and

$$
\begin{aligned}
& \sigma_{1}^{T}(s)=\left[\begin{array}{llll}
x^{T}(s) & x^{T}\left(s-\delta_{1}\right) & \cdots & x^{T}\left(s-(l-1) \delta_{1}\right)
\end{array}\right] \\
& h_{1}^{T}\left(\sigma_{1}(s)\right)=\left[\begin{array}{lllll}
f^{T}(x(s)) & f^{T}\left(x\left(s-\delta_{1}\right)\right) & \cdots & f^{T}\left(x\left(s-(l-1) \delta_{1}\right)\right)
\end{array}\right], \\
& \sigma_{2}^{T}(s)=\left[\begin{array}{lllll}
x^{T}(s) & x^{T}\left(s-\delta_{2}\right) & \cdots & x^{T}\left(s-(m-1) \delta_{2}\right)
\end{array}\right], \\
& h_{2}^{T}\left(\sigma_{2}(s)\right)=\left[\begin{array}{lllll}
f^{T}(x(s)) & f^{T}\left(x\left(s-\delta_{2}\right)\right) & \cdots & f^{T}\left(x\left(s-(m-1) \delta_{2}\right)\right)
\end{array}\right] .
\end{aligned}
$$

Denoting a parameter set $\Phi=\left\{P, Q, K, L, W_{1 i}, W_{2 h},\left[\begin{array}{cc}P_{1} & H_{1} \\ * & Q_{1}\end{array}\right],\left[\begin{array}{cc}\tilde{P}_{j} & \widetilde{H}_{j} \\ * & \widetilde{Q}_{j}\end{array}\right],\left[\begin{array}{cc}X_{j i} & Y_{j i} \\ * & Z_{j i}\end{array}\right],\left[\begin{array}{cc}X_{k h} & Y_{k h} \\ * & Z_{k h}\end{array}\right], j=\right.$ $1,2, i=1, \ldots, l ; k=3,4, h=1, \ldots, m\}$, then we give one proposition which is essential in the following deduction.

Proposition 3.1. If the parameter set $\Phi$ satisfies the following condition:

$$
\begin{aligned}
& P>0, \quad Q>0, \quad K>0, \quad L>0, \quad W_{1 i}>0, \quad W_{2 h}>0, \\
& {\left[\begin{array}{cc}
P_{1} & H_{1} \\
* & Q_{1}
\end{array}\right]>0, \quad\left[\begin{array}{cc}
\widetilde{P}_{j} & \widetilde{H}_{j} \\
* & \widetilde{Q}_{j}
\end{array}\right]>0, \quad\left[\begin{array}{cc}
X_{j i} & Y_{j i} \\
* & Z_{j i}
\end{array}\right]>0,} \\
& {\left[\begin{array}{cc}
X_{k h} & Y_{k h} \\
* & Z_{k h}
\end{array}\right]>0, \quad j=1,2, k=3,4 ; i=1, \ldots, l ; h=1, \ldots, m,}
\end{aligned}
$$

then the Lyapunov-Krasovskii functional (3.3) is definitely positive.

Moreover, in order to simplify the subsequent proof, we also give some notations in the following:

$$
\begin{aligned}
& \bar{\mu}_{1}=\mu_{2}-\mu_{1}, \quad \bar{\mu}_{3}=\mu_{4}-\mu_{3}, \quad \tilde{\Sigma}_{i}=\operatorname{diag}\left\{\Sigma_{i}, \ldots, \Sigma_{i}\right\}_{l n \times l n}, \\
& \bar{\Sigma}_{i}=\operatorname{diag}\left\{\Sigma_{i}, \ldots, \Sigma_{i}\right\}_{m n \times m n}, \quad \tilde{X}_{i}=\operatorname{diag}\left\{X_{i 1}, \ldots, X_{i l}\right\}, \\
& \tilde{Y}_{i}=\operatorname{diag}\left\{Y_{i 1}, \ldots, Y_{i l}\right\}, \quad \tilde{Z}_{i}=\operatorname{diag}\left\{Z_{i 1}, \ldots, Z_{i l}\right\}, \quad i=1,2, \\
& \tilde{S}_{1}=\operatorname{diag}\left\{S_{11}, \ldots, S_{1 l}\right\}, \quad \widetilde{W}_{1}=\operatorname{diag}\left\{W_{11}, \ldots, W_{1 l}\right\}, \\
& \tilde{V}_{1}=\operatorname{diag}\left\{V_{11}, \ldots, V_{1 l}\right\}, \quad \tilde{R}_{1}=\operatorname{diag}\left\{R_{11}, \ldots, R_{1 l}\right\},
\end{aligned}
$$


Discrete Dynamics in Nature and Society

$$
\begin{array}{ll}
\tilde{X}_{j}=\operatorname{diag}\left\{X_{j 1}, \ldots, X_{j m}\right\}, & \tilde{Y}_{j}=\operatorname{diag}\left\{Y_{j 1}, \ldots, Y_{j m}\right\}, \\
\tilde{Z}_{j}=\operatorname{diag}\left\{Z_{j 1}, \ldots, Z_{j m}\right\}, & j=3,4, \\
\tilde{S}_{2}=\operatorname{diag}\left\{S_{21}, \ldots, S_{2 m}\right\}, & \widetilde{W}_{2}=\operatorname{diag}\left\{W_{21}, \ldots, W_{2 m}\right\}, \\
\tilde{V}_{2}=\operatorname{diag}\left\{V_{21}, \ldots, V_{2 m}\right\}, & \widetilde{R}_{2}=\operatorname{diag}\left\{R_{21}, \ldots, R_{2 m}\right\} .
\end{array}
$$

Theorem 3.2. For two given positive integers $l, m$, and time-delay satisfying (2.2), the delayed neural network (3.1) is globally asymptotically stable in the mean square sense, if there exists one parameter set $\Phi$ satisfying Proposition 3.1, $n \times n$ matrices $E_{i}(i=1,2)$, and $n \times n$ diagonal matrices $U_{i}>0(i=$ $1,2,3,4,5), V_{1 i}>0, R_{1 i}>0, S_{1 i}(i=1, \ldots, l)$ making $\left[\begin{array}{cc}W_{1 i} & S_{1 i} \\ * & W_{1 i}\end{array}\right] \geq 0, V_{2 j}>0, R_{2 j}>0, S_{2 i}(j=$ $1, \ldots, m)$ making $\left[\begin{array}{cc}W_{2 i} & S_{2 i} \\ * & W_{2 i}\end{array}\right] \geq 0$ such that the following LMIs in (3.9) hold:

$$
\begin{aligned}
\Upsilon_{1} \Theta \Upsilon_{1}^{T} & +\Upsilon_{2} \Xi \Upsilon_{2}^{T}+\frac{\bar{\mu}_{1}}{l} \sum_{i=1}^{l} I_{1 i}^{T}\left[\begin{array}{cc}
X_{e i} & \Upsilon_{e i} \\
* & Z_{e i}
\end{array}\right] I_{1 i} \\
& +\frac{\bar{\mu}_{3}}{m} \sum_{i=1}^{m} I_{2 i}^{T}\left[\begin{array}{cc}
X_{f i} & \Upsilon_{f i} \\
* & Z_{f i}
\end{array}\right] I_{2 i}<0, \quad e=1,2 ; f=3,4,
\end{aligned}
$$

where

$$
\begin{aligned}
1 & I_{1 i}=\left[\begin{array}{ccccccccccc}
0_{n \cdot(m+l+1+i) n} & -I_{n} & 0_{n \cdot(2 l+2 m+1) n} & -I_{n} & * \\
0_{n \cdot(m+l+1+i) n} & I_{n} & 0_{n \cdot(2 l+2 m+1) n} & I_{n} & *
\end{array}\right], \\
& I_{2 i}=\left[\begin{array}{cccccccccccc}
0_{n \cdot(m+2 l+1+i) n} & -I_{n} & 0_{n \cdot(2 l+2 m+1) n} & -I_{n} & * \\
0_{n \cdot(m+2 l+1+i) n} & I_{n} & 0_{n \cdot(2 l+2 m+1) n} & I_{n} & *
\end{array}\right], \\
& =\left[\begin{array}{ccccccccccccc}
\Theta_{11} & Q & 0 & 0 & \Theta_{15} & 0 & 0 & 0 & \Theta_{19} & 0 & 0 & \Theta_{1,12} & \Theta_{1,13} \\
* & \Theta_{22} & 0 & 0 & 0 & -H_{1} & 0 & 0 & 0 & 0 & 0 & 0 & 0 \\
* & * & \Theta_{33} & 0 & 0 & 0 & U_{2} \Sigma_{2} & 0 & 0 & 0 & 0 & 0 & 0 \\
* & * & * & \Theta_{44} & 0 & 0 & 0 & U_{3} \Sigma_{2} & 0 & 0 & 0 & 0 & 0 \\
* & * & * & * & \Theta_{55} & 0 & 0 & 0 & \Theta_{59} & 0 & 0 & 0 & 0 \\
* & * & * & * & * & -Q_{1} & 0 & 0 & 0 & 0 & 0 & 0 & 0 \\
* & * & * & * & * & * & -U_{2} & 0 & 0 & 0 & 0 & 0 & 0 \\
* & * & * & * & * & * & * & -U_{3} & 0 & 0 & 0 & 0 \\
* & * & * & * & * & * & * & * & \Theta_{99} & 0 & 0 & \Theta_{9,12} & \Theta_{9,13} \\
* & * & * & * & * & * & * & * & * & -U_{4} \Sigma_{1} & 0 & U_{4} \Sigma_{2} & 0 \\
* & * & * & * & * & * & * & * & * & * & -U_{5} \Sigma_{1} & 0 & U_{5} \Sigma_{2} \\
* & * & * & * & * & * & * & * & * & * & * & -U_{4} & 0 \\
* & * & * & * & * & * & * & * & * & * & * & * & -U_{5}
\end{array}\right],
\end{aligned}
$$




$$
\Xi=\left[\begin{array}{cc}
\bar{\Xi}_{1} & \bar{\Xi}_{2} \\
* & \bar{\Xi}_{3}
\end{array}\right], \quad \bar{\Xi}_{1}=\left[\begin{array}{cccccc}
\Xi_{11} & 0 & 0 & 0 & \widetilde{S}_{1} & 0 \\
* & \Xi_{22} & 0 & 0 & \widetilde{S}_{1} & 0 \\
* & * & \Xi_{33} & 0 & 0 & \widetilde{S}_{2} \\
* & * & * & \Xi_{44} & 0 & \widetilde{S}_{2} \\
* & * & * & * & \Xi_{55} & 0 \\
* & * & * & * & * & \Xi_{66}
\end{array}\right],
$$

$\bar{\Xi}_{2}=\operatorname{diag}\left\{\Xi_{17}, \Xi_{28}, \Xi_{39}, \Xi_{4,10}, \Xi_{5,11}, \Xi_{6,12}\right\}, \quad \bar{\Xi}_{3}=\operatorname{diag}\left\{\Xi_{77}, \Xi_{88}, \Xi_{99}, \Xi_{10,10}, \Xi_{11,11}, \Xi_{12,12}\right\}$,

$$
\Upsilon_{1}=\left[\begin{array}{ccc}
I_{n} & & * \\
0_{n \cdot n} & I_{n} & * \\
0_{n \cdot(l+1) n} & I_{n} & * \\
0_{n \cdot(m+l+1) n} & I_{n} & * \\
0_{n \cdot 2(m+l+1) n} & I_{n} & * \\
0_{n \cdot(2 m+2 l+3) n} & I_{n} & * \\
0_{n \cdot(2 m+3 l+3) n} & I_{n} & * \\
0_{n \cdot 3(m+l+1) n} & I_{n} & * \\
0_{n \cdot 4(m+l+1) n} & I_{n} & * \\
0_{n \cdot(4 m+4 l+5) n} & I_{n} & * \\
0_{n \cdot(4 m+4 l+6) n} & I_{n} & * \\
0_{n \cdot(4 m+4 l+7) n} & I_{n} & * \\
0_{n \cdot(4 m+4 l+8) n} & & I_{n}
\end{array}\right], \quad \Upsilon_{2}=\left[\begin{array}{ccc}
0_{l n \cdot n} & I_{l n} * \\
0_{l n \cdot 2 n} & I_{l n} * \\
0_{m n \cdot(l+1) n} & I_{m n} * \\
0_{m n \cdot(l+2) n} & I_{m n} * \\
0_{l n \cdot(m+l+2) n} & I_{l n} * \\
0_{m n \cdot(m+2 l+2) n} & I_{m n} * \\
0_{l n \cdot(2 m+2 l+3) n} & I_{l n} * \\
0_{l n \cdot(2 m+2 l+4) n} & I_{l n} * \\
0_{m n \cdot(2 m+3 l+3) n} & I_{m n} * \\
0_{m n \cdot(2 m+3 l+4) n} & I_{m n} * \\
0_{l n \cdot(3 m+3 l+4) n} & I_{l n} * \\
0_{m n \cdot(3 m+4 l+4) n} & I_{m n} *
\end{array}\right],
$$

with the notations $*$ in $I_{j i}(j=1, \ldots, 6)$ and $\Upsilon_{g}(g=1,2)$ denoting the appropriately dimensional zero matrices making them $(4 l+4 m+9) n$ columns:

$$
\begin{aligned}
& \Theta_{11}=-E_{1}^{T} C-C^{T} E_{1}+P_{1}-Q-U_{1} \Sigma_{1}, \quad \Theta_{15}=E_{1}^{T} A+H_{1}+U_{1} \Sigma_{2}, \\
& \Theta_{19}=P-\Sigma K+\bar{\Sigma} L-E_{1}^{T}-C^{T} E_{2}, \quad \Theta_{1,12}=\delta_{0} E_{1}^{T} B \\
& \Theta_{1,13}=\left(1-\delta_{0}\right) E_{1}^{T} B, \quad \Theta_{22}=-P_{1}-Q, \quad \Theta_{33}=-U_{2} \Sigma_{1}, \\
& \Theta_{44}=-U_{3} \Sigma_{1}, \quad \Theta_{55}=-U_{1}+Q_{1}, \quad \Theta_{59}=K-L+A^{T} E_{2}, \\
& \Theta_{99}=-E_{2}^{T}-E_{2}+\tau_{1}^{2} Q+\sum_{i=1}^{l} \delta_{1}^{2} W_{1 i}+\sum_{i=1}^{m} \delta_{2}^{2} W_{2 i}, \\
& \Theta_{9,12}=\delta_{0} E_{2}^{T} B, \quad \Theta_{9,13}=\left(1-\delta_{0}\right) E_{2}^{T} B \\
& \Xi_{11}=\widetilde{P}_{1}+\tilde{X}_{1}-\widetilde{W}_{1}-\widetilde{V}_{1} \widetilde{\Sigma}_{1}, \quad \Xi_{22}=-\widetilde{P}_{1}-\tilde{X}_{2}-\widetilde{W}_{1} \\
& \Xi_{33}=\widetilde{P}_{2}+\tilde{X}_{3}-\widetilde{W}_{2}-\tilde{V}_{2} \bar{\Sigma}_{1}, \quad \Xi_{44}=-\widetilde{P}_{2}-\widetilde{X}_{4}-\widetilde{W}_{2} \\
& \Xi_{55}=\left(1-\frac{\mu_{2}}{l}\right) \tilde{X}_{2}-\left(1-\frac{\mu_{1}}{l}\right) \tilde{X}_{1}-2 \widetilde{W}_{1}-\widetilde{R}_{1} \widetilde{\Sigma}_{1}, \\
& \Xi_{66}=\left(1-\frac{\mu_{4}}{m}\right) \tilde{X}_{4}-\left(1-\frac{\mu_{3}}{m}\right) \tilde{X}_{3}-2 \widetilde{W}_{2}-\widetilde{R}_{2} \bar{\Sigma}_{1},
\end{aligned}
$$


Discrete Dynamics in Nature and Society

$$
\begin{array}{lc}
\Xi_{77}=\widetilde{Q}_{1}+\tilde{Z}_{1}-\tilde{V}_{1}, \quad \Xi_{88}=-\widetilde{Q}_{1}-\tilde{Z}_{2}, & \Xi_{99}=-\tilde{V}_{2}+\widetilde{Q}_{2}+\tilde{Z}_{3}, \quad \Xi_{10,10}=-\widetilde{Q}_{2}-\tilde{Z}_{4}, \\
\Xi_{11,11}=\left(1-\frac{\mu_{2}}{l}\right) \tilde{Z}_{2}-\left(1-\frac{\mu_{1}}{l}\right) \tilde{Z}_{1}-\widetilde{R}_{1}, & \Xi_{12,12}=\left(1-\frac{\mu_{4}}{m}\right) \tilde{Z}_{4}-\left(1-\frac{\mu_{3}}{m}\right) \tilde{Z}_{3}-\widetilde{R}_{2}, \\
\Xi_{17}=\widetilde{H}_{1}+\tilde{Y}_{1}+\tilde{V}_{1} \tilde{\Sigma}_{2}, \quad \Xi_{28}=-\widetilde{H}_{1}-\tilde{Y}_{2}, & \Xi_{39}=\widetilde{H}_{2}+\tilde{Y}_{3}+\tilde{V}_{2} \bar{\Sigma}_{2}, \quad \Xi_{4,10}=-\widetilde{H}_{2}-\tilde{Y}_{4}, \\
\Xi_{5,11}=\left(1-\frac{\mu_{2}}{l}\right) \tilde{Y}_{2}-\left(1-\frac{\mu_{1}}{l}\right) \tilde{Y}_{1}+\widetilde{R}_{1} \widetilde{\Sigma}_{1}, & \Xi_{6,12}=\left(1-\frac{\mu_{4}}{l}\right) \tilde{Y}_{4}-\left(1-\frac{\mu_{3}}{l}\right) \tilde{Y}_{3}+\widetilde{R}_{2} \bar{\Sigma}_{2} .
\end{array}
$$

Proof. Firstly, we show the uniqueness of the equilibrium point by the method of contradiction. Here we can denote the equilibrium point as $z^{*}$ of DNNs (2.9), then we have

$$
\begin{aligned}
0 & =-C z^{*}+A f\left(z^{*}\right)+\delta(t) B f\left(z^{*}\right)+(1-\delta(t)) B f\left(z^{*}\right) \\
& =-C z^{*}+(A+B) f\left(z^{*}\right) .
\end{aligned}
$$

Now we suppose that the other equilibrium point $z^{*} \neq 0$ exists, then it follows that

$$
\begin{gathered}
2\left(z^{*}\right)^{T} E_{1}^{T}\left[-C z^{*}+(A+B) f\left(z^{*}\right)\right]=0, \\
-\left(z^{*}\right)^{T} U_{1} \Sigma_{1} z^{*}+2\left(z^{*}\right)^{T} U_{1} \Sigma_{2} f\left(z^{*}\right)-f^{T}\left(z^{*}\right) U_{1} f\left(z^{*}\right) \geq 0 .
\end{gathered}
$$

Then combining (3.13) and (3.14) yields that

$$
\left[\begin{array}{c}
z^{*} \\
f\left(z^{*}\right)
\end{array}\right]^{T}\left[\begin{array}{cc}
\Pi_{11} & \Pi_{12} \\
\Pi_{12}^{T} & \Pi_{22}
\end{array}\right]\left[\begin{array}{c}
z^{*} \\
f\left(z^{*}\right)
\end{array}\right] \geq 0
$$

with $\Pi_{11}=-E_{1}^{T} C-C^{T} E_{1}-U_{1} \Sigma_{1}, \Pi_{12}=E_{1}^{T}(A+B)+U_{1} \Sigma_{2}$, and $\Pi_{22}=-U_{1}$. Meanwhile, it is noted that

$$
\left[\begin{array}{c}
z^{*} \\
f\left(z^{*}\right)
\end{array}\right]^{T}\left[\begin{array}{cc}
P_{1} & H_{1} \\
* & Q_{1}
\end{array}\right]\left[\begin{array}{c}
z^{*} \\
f\left(z^{*}\right)
\end{array}\right] \geq 0
$$

Yet on the other hand, let

$$
\Psi=\left[\begin{array}{ccccc}
I_{n} & I_{n} & 0_{n \times(4 l+4 m+7) n} & \\
0_{n \times(2 l+2 m+2) n} & I_{n} & 0_{n \times(2 l+2 m+4) n} & I_{n} & I_{n}
\end{array}\right] .
$$

Then multiplying the term $\Upsilon_{1} \Theta \Upsilon_{1}^{T}+\Upsilon_{2} \Xi \Upsilon_{2}^{T}$ in (3.9) by $\Psi$ and $\Psi^{T}$ on its left hand and right one, respectively, and using (3.15) and (3.16), we can deduce that

$$
\left[\begin{array}{cc}
\Pi_{11}+P_{1} & \Pi_{12}+H_{1} \\
\Pi_{12}^{T}+H_{1}^{T} & \Pi_{22}+Q_{1}
\end{array}\right]<0 \Longrightarrow \Pi=\left[\begin{array}{ll}
\Pi_{11} & \Pi_{12} \\
\Pi_{12}^{T} & \Pi_{22}
\end{array}\right]<-\left[\begin{array}{cc}
P_{1} & H_{1} \\
H_{1}^{T} & Q_{1}
\end{array}\right]
$$


Thus we can derive $\Pi<0$, which contradicts with (3.15) and implies $z^{*}=0$. This is to say, the origin of the DNNs (3.1) is the unique equilibrium point.

Next, through directly calculating and using the denotations in (3.7)-(3.8), the stochastic differential of $V_{i}\left(x_{t}\right)(i=1,2)$ in (3.3) along the trajectories of system (3.1) yields

$$
\begin{aligned}
& \mathcal{L} V_{1}\left(x_{t}\right)=2 x^{T}(t) P \dot{x}(t)+\dot{x}^{T}(t) \tau_{1}^{2} Q \dot{x}(t)-\int_{t-\tau_{1}}^{t} \tau_{1} \dot{x}^{T}(s) Q \dot{x}(s) d s \\
& +2\left[f^{T}(x(t))(K-L)+x^{T}(t)(\bar{\Sigma} L-\Sigma K)\right] \dot{x}(t) \\
& \leq 2 x^{T}(t) P \dot{x}(t)+\dot{x}^{T}(t) \tau_{1}^{2} Q \dot{x}(t)-\left[x(t)-x\left(t-\tau_{1}\right)\right]^{T} Q\left[x(t)-x\left(t-\tau_{1}\right)\right] \\
& +2\left[f^{T}(x(t))(K-L)+x^{T}(t)(\bar{\Sigma} L-\Sigma K)\right] \dot{x}(t), \\
& \mathcal{L} V_{2}\left(x_{t}\right)=\left[x^{T}(t) P_{1} x(t)+2 x^{T}(t) H_{1} f(x(t))+f^{T}(x(t)) Q_{1} f(x(t))\right] \\
& -\left[x^{T}\left(t-\tau_{1}\right) P_{1} x\left(t-\tau_{1}\right)+2 x^{T}\left(t-\tau_{1}\right) H_{1} f\left(x\left(t-\tau_{1}\right)\right)+f^{T}\left(x\left(t-\tau_{1}\right)\right) Q_{1} f\left(x\left(t-\tau_{1}\right)\right)\right] \\
& +\left[\sigma_{1}^{T}\left(t-\tau_{1}\right) \tilde{P}_{1} \sigma_{1}\left(t-\tau_{1}\right)+2 \sigma_{1}^{T}\left(t-\tau_{1}\right) \widetilde{H}_{1} h_{1}\left(\sigma_{1}\left(t-\tau_{1}\right)\right)\right. \\
& \left.+h_{1}^{T}\left(\sigma_{1}\left(t-\tau_{1}\right)\right) \widetilde{Q}_{1} h_{1}\left(\sigma_{1}\left(t-\tau_{1}\right)\right)\right] \\
& -\left[\sigma_{1}^{T}\left(t-\tau_{1}-\delta_{1}\right) \widetilde{P}_{1} \times \sigma\left(t-\tau_{1}-\delta_{1}\right)+2 \sigma_{1}^{T}\left(t-\tau_{1}-\delta_{1}\right) \widetilde{H}_{1} h_{1}\left(\sigma\left(t-\tau_{1}-\delta_{1}\right)\right)\right. \\
& \left.+h_{1}^{T}\left(\sigma\left(t-\tau_{1}-\delta_{1}\right)\right) \tilde{Q}_{1} \times h_{1}\left(\sigma\left(t-\tau_{1}-\delta_{1}\right)\right)\right] \\
& +\left[\sigma_{2}^{T}\left(t-\tau_{2}\right) \tilde{P}_{2} \sigma_{2}\left(t-\tau_{2}\right)+2 \sigma_{2}^{T}\left(t-\tau_{2}\right) \widetilde{H}_{2} h_{2}\left(\sigma_{2}\left(t-\tau_{2}\right)\right)\right. \\
& \left.+h_{2}^{T}\left(\sigma_{2}\left(t-\tau_{2}\right)\right) \widetilde{Q}_{2} h_{2}\left(\sigma_{2}\left(t-\tau_{2}\right)\right)\right] \\
& -\left[\sigma_{2}^{T}\left(t-\tau_{2}-\delta_{2}\right) \widetilde{P}_{2} \sigma\left(t-\tau_{2}-\delta_{2}\right)+2 \sigma_{2}^{T}\left(t-\tau_{2}-\delta_{2}\right) \widetilde{H}_{2} h_{2}\left(\sigma\left(t-\tau_{2}-\delta_{2}\right)\right)\right. \\
& \left.+h_{2}^{T}\left(\sigma\left(t-\tau_{2}-\delta_{2}\right)\right) \widetilde{Q}_{2} h_{2}\left(\sigma\left(t-\tau_{2}-\delta_{2}\right)\right)\right] \\
& +\left[\sigma_{1}^{T}\left(t-\tau_{1}\right) \tilde{X}_{1} \sigma_{1}\left(t-\tau_{1}\right)+\sigma_{1}^{T}\left(t-\tau_{1}-\rho_{1}(t)\right)\left(1-\frac{\dot{\tau}_{1}(t)}{l}\right)\left(\tilde{X}_{2}-\tilde{X}_{1}\right)\right. \\
& \left.\times \sigma_{1}\left(t-\tau_{1}-\rho_{1}(t)\right)-\sigma_{1}^{T}\left(t-\tau_{1}-\delta_{1}\right) \tilde{X}_{2} \sigma_{1}\left(t-\tau_{1}-\delta_{1}\right)\right] \\
& +\left[2 \sigma_{1}^{T}\left(t-\tau_{1}\right) \tilde{Y}_{1} h_{1}\left(\sigma_{1}\left(t-\tau_{1}\right)\right)+2 \sigma_{1}^{T}\left(t-\tau_{1}-\rho_{1}(t)\right) \times\left(1-\frac{\dot{\tau}_{1}(t)}{l}\right)\left(\tilde{Y}_{2}-\tilde{Y}_{1}\right)\right. \\
& \left.\times h_{1}\left(\sigma_{1}\left(t-\tau_{1}-\rho_{1}(t)\right)\right)-2 \sigma_{1}^{T}\left(t-\tau_{1}-\delta_{1}\right) \tilde{Y}_{2} h_{1}\left(\sigma_{1}\left(t-\tau_{1}-\delta_{1}\right)\right)\right] \\
& +\left[h_{1}^{T}\left(\sigma_{1}\left(t-\tau_{1}\right)\right) \tilde{Z}_{1} h_{1}\left(\sigma_{1}\left(t-\tau_{1}\right)\right)+h_{1}^{T}\left(\sigma_{1}\left(t-\tau_{1}-\rho_{1}(t)\right)\right)\left(1-\frac{\dot{\tau}_{1}(t)}{l}\right)\right.
\end{aligned}
$$


Discrete Dynamics in Nature and Society

$$
\begin{aligned}
& \left.\times\left(\tilde{Z}_{2}-\tilde{Z}_{1}\right) h_{1}\left(\sigma_{1}\left(t-\tau_{1}-\rho_{1}(t)\right)\right)-h_{1}^{T}\left(\sigma_{1}\left(t-\tau_{1}-\delta_{1}\right)\right) \tilde{Z}_{2} h_{1}^{T}\left(\sigma_{1}\left(t-\tau_{1}-\delta_{1}\right)\right)\right] \\
+ & {\left[\sigma_{2}^{T}\left(t-\tau_{2}\right) \tilde{X}_{3} \sigma_{2}\left(t-\tau_{2}\right)+\sigma_{2}^{T}\left(t-\tau_{2}-\rho_{2}(t)\right)\left(1-\frac{\dot{\tau}_{2}(t)}{m}\right)\left(\tilde{X}_{4}-\tilde{X}_{3}\right)\right.} \\
& \left.\times \sigma_{2}\left(t-\tau_{2}-\rho_{2}(t)\right)-\sigma_{2}^{T}\left(t-\tau_{2}-\delta_{2}\right) \tilde{X}_{4} \sigma_{2}\left(t-\tau_{2}-\delta_{2}\right)\right] \\
+ & {\left[2 \sigma_{2}^{T}\left(t-\tau_{2}\right) \tilde{Y}_{3} h_{2}\left(\sigma_{2}\left(t-\tau_{2}\right)\right)+2 \sigma_{2}^{T}\left(t-\tau_{2}-\rho_{2}(t)\right) \times\left(1-\frac{\dot{\tau}_{2}(t)}{m}\right)\right.} \\
& \left.\times\left(\tilde{Y}_{4}-\tilde{Y}_{3}\right) h_{2}\left(\sigma_{2}\left(t-\tau_{2}-\rho_{2}(t)\right)\right)-2 \sigma_{2}^{T}\left(t-\tau_{2}-\delta_{2}\right) \tilde{Y}_{4} h_{2}\left(\sigma_{2}\left(t-\tau_{2}-\delta_{2}\right)\right)\right] \\
+ & {\left[h_{2}^{T}\left(\sigma_{2}\left(t-\tau_{2}\right)\right) \tilde{Z}_{3} h_{2}\left(\sigma_{2}\left(t-\tau_{2}\right)\right)+h_{2}^{T}\left(\sigma_{2}\left(t-\tau_{2}-\rho_{2}(t)\right)\right)\left(1-\frac{\dot{\tau}_{2}(t)}{m}\right)\right.} \\
& \left.\times\left(\tilde{Z}_{4}-\tilde{Z}_{3}\right) h_{2}\left(\sigma_{2}\left(t-\tau_{2}-\rho_{2}(t)\right)\right)-h_{2}^{T}\left(\sigma_{2}\left(t-\tau_{2}-\delta_{2}\right)\right) \tilde{Z}_{4} h^{T}\left(\sigma_{2}\left(t-\tau_{2}-\delta_{2}\right)\right)\right] .
\end{aligned}
$$

Moreover, we can compute out $\mathcal{L} V_{3}\left(x_{t}\right)$ as follows:

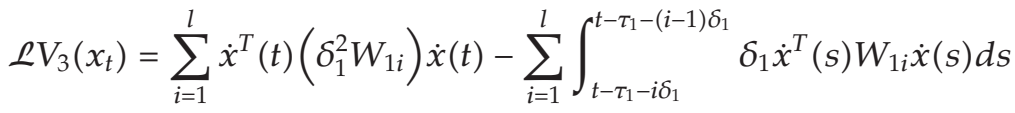

$$
\begin{aligned}
& +\sum_{i=1}^{m} \dot{x}^{T}(t)\left(\delta_{2}^{2} W_{2 i}\right) \dot{x}(t) \\
& -\sum_{i=1}^{m} \int_{t-\tau_{2}-i \delta_{2}}^{t-\tau_{2}-(i-1) \delta_{2}} \dot{x}^{T}(s)\left(\delta_{2} W_{2 i}\right) \dot{x}(s) d s .
\end{aligned}
$$

Then, by resorting to Lemmas 2.4 and $2.5\left[\begin{array}{cc}W_{1 i} & S_{1 i} \\ * & W_{1 i}\end{array}\right] \geq 0,\left[\begin{array}{cc}W_{2 i} & S_{2 i} \\ * & W_{2 i}\end{array}\right] \geq 0$, and using the donations $\widetilde{W}_{i}, \widetilde{S}_{i}(i=1,2)$, the following inequalities can be derived:

$$
\begin{aligned}
-\sum_{i=1}^{l} \int_{t-\tau_{1}-i \delta_{1}}^{t-\tau_{1}-(i-1) \delta_{1}} \delta_{1} \dot{x}^{T}(s) W_{1 i} \dot{x}(s) d s \\
\leq \sum_{i=1}^{l}\left[-\left[x\left(t-\tau_{1}-(i-1) \delta_{1}-\rho_{1}(t)\right)-x\left(t-\tau_{1}-i \delta_{1}\right)\right]^{T} W_{1 i}\right. \\
\times\left[x\left(t-\tau_{1}-(i-1) \delta_{1}-\rho_{1}(t)\right)-x\left(t-\tau_{1}-i \delta_{1}\right)\right] \\
-2\left[x\left(t-\tau_{1}-(i-1) \delta_{1}-\rho_{1}(t)\right)-x\left(t-\tau_{1}-i \delta_{1}\right)\right]^{T} S_{1 i} \\
\times\left[x\left(t-\tau_{1}-(i-1) \delta_{1}\right)-x\left(t-\tau_{1}-(i-1) \delta_{1}-\rho_{1}(t)\right)\right]
\end{aligned}
$$




$$
\begin{array}{r}
-\left[x\left(t-\tau_{1}-(i-1) \delta_{1}\right)-x\left(t-\tau_{1}-(i-1) \delta_{1}-\rho_{1}(t)\right)\right]^{T} W_{1 i} \\
\left.\times\left[x\left(t-\tau_{1}-(i-1) \delta_{1}\right)-x\left(t-\tau_{1}-(i-1) \delta_{1}-\rho_{1}(t)\right)\right]\right] \\
=-\left[\sigma_{1}^{T}\left(t-\tau_{1}-\rho_{1}(t)\right) \widetilde{W}_{1} \sigma\left(t-\tau_{1}-\rho_{1}(t)\right)-2 \sigma_{1}^{T}\left(t-\tau_{1}-\rho_{1}(t)\right)\right. \\
\left.\times \widetilde{S}_{1} \sigma_{1}\left(t-\tau_{1}-\delta_{1}\right)+\sigma_{1}^{T}\left(t-\tau_{1}-\delta_{1}\right) \widetilde{W}_{1} \sigma_{1}\left(t-\tau_{1}-\delta_{1}\right)\right] \\
-\left[\sigma_{1}^{T}\left(t-\tau_{1}-\rho_{1}(t)\right) \widetilde{W}_{1} \sigma_{1}\left(t-\tau_{1}-\rho_{1}(t)\right)\right. \\
\left.-2 \sigma_{2}^{T}\left(t-\tau_{2}-\rho_{2}(t)\right) \widetilde{S}_{1} \sigma_{2}\left(t-\tau_{2}\right)+\sigma_{2}^{T}\left(t-\tau_{2}\right) \widetilde{W}_{2} \sigma_{2}\left(t-\tau_{2}\right)\right], \\
-\sum_{i=1}^{m} \int_{t-\tau_{2}-i \delta_{2}}^{t-\tau_{2}-(i-1) \delta_{2}} \delta_{2} \dot{x}^{T}(s) W_{2 i} \dot{x}(s) d s \\
\leq-\left[\sigma_{2}^{T}\left(t-\tau_{2}-\rho_{2}(t)\right) \widetilde{W}_{2} \sigma\left(t-\tau_{2}-\rho_{2}(t)\right)-2 \sigma_{2}^{T}\left(t-\tau_{2}-\rho_{2}(t)\right)\right. \\
\left.\times \widetilde{S}_{2} \sigma_{2}\left(t-\tau_{2}-\delta_{2}\right)+\sigma_{2}^{T}\left(t-\tau_{2}-\delta_{2}\right) \widetilde{W}_{2} \sigma_{2}\left(t-\tau_{2}-\delta_{2}\right)\right] \\
-\left[\sigma_{2}^{T}\left(t-\tau_{2}-\rho_{2}(t)\right) \widetilde{W}_{2} \sigma_{2}\left(t-\tau_{2}-\rho_{2}(t)\right)\right. \\
\left.-2 \sigma_{2}^{T}\left(t-\tau_{2}-\rho_{2}(t)\right) \widetilde{S}_{2} \sigma_{2}\left(t-\tau_{2}\right)+\sigma_{2}^{T}\left(t-\tau_{2}\right) \widetilde{W}_{2} \sigma_{2}\left(t-\tau_{2}\right)\right] .
\end{array}
$$

Then, it follows from (3.21) that $\mathcal{L} V_{3}\left(x_{t}\right)$ satisfies

$$
\begin{aligned}
& \mathcal{L} V_{3}\left(x_{t}\right) \leq \sum_{i=1}^{l} \dot{x}^{T}(t)\left(\delta_{1}^{2} W_{1 i}\right) \dot{x}(t)+\sum_{i=1}^{l} \dot{x}^{T}(t)\left(\delta_{2}^{2} W_{2 i}\right) \dot{x}(t) \\
&-\left[\sigma_{1}^{T}\left(t-\tau_{1}-\rho_{1}(t)\right) \widetilde{W}_{1} \sigma\left(t-\tau_{1}-\rho_{1}(t)\right)\right. \\
&-\left.2 \sigma_{1}^{T}\left(t-\tau_{1}-\rho_{1}(t)\right) \widetilde{S}_{1} \sigma_{1}\left(t-\tau_{1}-\delta_{1}\right)+\sigma_{1}^{T}\left(t-\tau_{1}-\delta_{1}\right) \widetilde{W}_{1} \sigma_{1}\left(t-\tau_{1}-\delta_{1}\right)\right] \\
&- {\left[\sigma_{1}^{T}\left(t-\tau_{1}-\rho_{1}(t)\right) \widetilde{W}_{1} \sigma_{1}\left(t-\tau_{1}-\rho_{1}(t)\right)-2 \sigma_{2}^{T}\left(t-\tau_{2}-\rho_{2}(t)\right) \widetilde{S}_{1} \sigma_{2}\left(t-\tau_{2}\right)\right.} \\
&\left.+\sigma_{2}^{T}\left(t-\tau_{2}\right) \widetilde{W}_{1} \sigma_{2}\left(t-\tau_{2}\right)\right] \\
&- {\left[\sigma_{2}^{T}\left(t-\tau_{2}-\rho_{2}(t)\right) \widetilde{W}_{2} \sigma\left(t-\tau_{2}-\rho_{2}(t)\right)-2 \sigma_{2}^{T}\left(t-\tau_{2}-\rho_{2}(t)\right) \times \widetilde{S}_{2} \sigma_{2}\left(t-\tau_{2}-\delta_{2}\right)\right.} \\
&\left.+\sigma_{2}^{T}\left(t-\tau_{2}-\delta_{2}\right) \widetilde{W}_{2} \sigma_{2}\left(t-\tau_{2}-\delta_{2}\right)\right] \\
&- {\left[\sigma_{2}^{T}\left(t-\tau_{2}-\rho_{2}(t)\right) \times \widetilde{W}_{2} \sigma_{2}\left(t-\tau_{2}-\rho_{2}(t)\right)\right.} \\
&\left.-2 \sigma_{2}^{T}\left(t-\tau_{2}-\rho_{2}(t)\right) \widetilde{S}_{2} \sigma_{2}\left(t-\tau_{2}\right)+\sigma_{2}^{T}\left(t-\tau_{2}\right) \widetilde{W}_{2} \sigma_{2}\left(t-\tau_{2}\right)\right] .
\end{aligned}
$$


From (2.10), for any $n \times n$ diagonal matrices $U_{i}>0(i=1,2,3,4,5), V_{1 i}>0, R_{1 i}>0$ (i= $1, \ldots, l), V_{2 j}>0, R_{2 j}>0(j=1, \ldots, m)$, and setting $\tilde{V}_{1}, \widetilde{R}_{1}$ in (3.7), $\tilde{V}_{2}, \widetilde{R}_{2}$ in (3.8), the following inequality holds:

$$
\begin{aligned}
& 0 \leq\left[-x^{T}(t) U_{1} \Sigma_{1} x(t)+2 x^{T}(t) U_{1} \Sigma_{2} f(x(t))-f^{T}(x(t)) U_{1} f(x(t))\right] \\
& +\left[-x^{T}\left(t-\tau_{2}\right) U_{2} \Sigma_{1} x\left(t-\tau_{2}\right)+2 x^{T}\left(t-\tau_{2}\right) U_{2} \Sigma_{2} f\left(x\left(t-\tau_{2}\right)\right)\right. \\
& \left.-f^{T}\left(x\left(t-\tau_{2}\right)\right) U_{2} f\left(x\left(t-\tau_{2}\right)\right)\right] \\
& +\left[-x^{T}\left(t-\tau_{3}\right) U_{3} \Sigma_{1} x\left(t-\tau_{3}\right)+2 x^{T}\left(t-\tau_{3}\right)\right. \\
& \left.\times U_{3} \Sigma_{2} f\left(x\left(t-\tau_{3}\right)\right)-f^{T}\left(x\left(t-\tau_{3}\right)\right) U_{3} f\left(x\left(t-\tau_{3}\right)\right)\right] \\
& +\left[-x^{T}\left(t-\tau_{1}(t)\right) \times U_{4} \Sigma_{1} x\left(t-\tau_{1}(t)\right)+2 x^{T}\left(t-\tau_{1}(t)\right) U_{4} \Sigma_{2} f\left(x\left(t-\tau_{1}(t)\right)\right)\right. \\
& \left.-f^{T}\left(x\left(t-\tau_{1}(t)\right)\right) U_{4} f\left(x\left(t-\tau_{1}(t)\right)\right)\right] \\
& +\left[-x^{T}\left(t-\tau_{2}(t)\right) U_{5} \Sigma_{1} x\left(t-\tau_{2}(t)\right)+2 x^{T}\left(t-\tau_{2}(t)\right) U_{5} \Sigma_{2} f\left(x\left(t-\tau_{2}(t)\right)\right)\right. \\
& \left.-f^{T}\left(x\left(t-\tau_{2}(t)\right)\right) U_{5} f\left(x\left(t-\tau_{2}(t)\right)\right)\right] \\
& +\left[-\sigma_{1}^{T}\left(t-\tau_{1}\right) \tilde{V}_{1} \tilde{\Sigma}_{1} \sigma_{1}\left(t-\tau_{1}\right)+2 \sigma_{1}^{T}\left(t-\tau_{1}\right) \tilde{V}_{1} \tilde{\Sigma}_{2} h_{1}\left(\sigma_{1}\left(t-\tau_{1}\right)\right)\right. \\
& \left.-h_{1}^{T}\left(\sigma_{1}\left(t-\tau_{1}\right)\right) \tilde{V}_{1} h_{1}\left(\sigma_{1}\left(t-\tau_{1}\right)\right)\right] \\
& +\left[-\sigma_{1}^{T}\left(t-\tau_{1}-\rho_{1}(t)\right) \tilde{R}_{1} \tilde{\Sigma}_{1} \sigma_{1}\left(t-\tau_{1}-\rho_{1}(t)\right)\right. \\
& +2 \sigma_{1}^{T}\left(t-\tau_{1}-\rho_{1}(t)\right) \tilde{R}_{1} \tilde{\Sigma}_{2} h_{1}\left(\sigma_{1}\left(t-\tau_{1}-\rho_{1}(t)\right)\right) \\
& \left.-h_{1}^{T}\left(\sigma_{1}\left(t-\tau_{1}-\rho_{1}(t)\right)\right) \tilde{R}_{1} h_{1}\left(\sigma_{1}\left(t-\tau_{1}-\rho_{1}(t)\right)\right)\right] \\
& +\left[-\sigma_{2}^{T}\left(t-\tau_{2}\right) \tilde{V}_{2} \bar{\Sigma}_{1} \sigma_{2}\left(t-\tau_{2}\right)+2 \sigma_{2}^{T}\left(t-\tau_{2}\right) \tilde{V}_{2} \bar{\Sigma}_{2} h_{2}\left(\sigma_{2}\left(t-\tau_{2}\right)\right)\right. \\
& \left.-h_{2}^{T}\left(\sigma_{2}\left(t-\tau_{2}\right)\right) \times \tilde{V}_{2} h_{2}\left(\sigma_{2}\left(t-\tau_{2}\right)\right)\right] \\
& +\left[-\sigma_{2}^{T}\left(t-\tau_{2}-\rho_{2}(t)\right) \tilde{R}_{2} \bar{\Sigma}_{1} \sigma_{2}\left(t-\tau_{2}-\rho_{2}(t)\right)\right. \\
& +2 \sigma_{2}^{T}\left(t-\tau_{2}-\rho_{2}(t)\right) \times \widetilde{R}_{2} \bar{\Sigma}_{2} h_{2}\left(\sigma_{2}\left(t-\tau_{2}-\rho_{2}(t)\right)\right) \\
& \left.-h_{2}^{T}\left(\sigma_{2}\left(t-\tau_{2}-\rho_{2}(t)\right)\right) \tilde{R}_{2} h_{2}\left(\sigma_{2}\left(t-\tau_{2}-\rho_{2}(t)\right)\right)\right] .
\end{aligned}
$$


Moreover, together with (3.1) and any $n \times n$ constant matrices $E_{1}, E_{2}$, one can deduce

$$
\begin{aligned}
0= & {\left[x^{T}(t) E_{1}^{T}+\dot{x}^{T}(t) E_{2}^{T}\right] } \\
& \times\left[-\dot{x}(t)-C x(t)+A f(x(t))+\delta_{0} B f\left(x\left(t-\tau_{1}(t)\right)\right)+\left(1-\delta_{0}\right) B f\left(x\left(t-\tau_{2}(t)\right)\right)\right. \\
& \left.\quad+\left(\delta(t)-\delta_{0}\right) B\left[f\left(x\left(t-\tau_{1}(t)\right)\right)-f\left(x\left(t-\tau_{2}(t)\right)\right)\right]\right] .
\end{aligned}
$$

Now adding the right terms (3.19) and (3.22)-(3.24) to $\mathcal{L V}\left(x_{t}\right)$ and taking the mathematical expectation on its both sides, we can deduce

$$
\begin{aligned}
& \mathrm{E}\left\{\mathcal{L} V\left(x_{t}\right)\right\} \leq \zeta^{T}(t)\left\{\Upsilon_{1} \Theta \Upsilon_{1}^{T}+\frac{\dot{\tau}_{1}(t)-\mu_{1}}{l} \sum_{i=1}^{l} \mathrm{I}_{1 i}^{T}\left[\begin{array}{cc}
X_{1 i} & \Upsilon_{1 i} \\
* & Z_{1 i}
\end{array}\right] \mathrm{I}_{1 i}\right. \\
& +\frac{\mu_{2}-\dot{\tau}_{1}(t)}{l} \sum_{i=1}^{l} \mathrm{I}_{1 i}^{T}\left[\begin{array}{cc}
X_{2 i} & Y_{2 i} \\
* & Z_{2 i}
\end{array}\right] \mathrm{I}_{1 i} \\
& +\Upsilon_{2} \Xi \Upsilon_{2}^{T}+\frac{\dot{\tau}_{2}(t)-\mu_{3}}{m} \sum_{i=1}^{m} \mathrm{I}_{2 i}^{T}\left[\begin{array}{cc}
X_{3 i} & \Upsilon_{3 i} \\
* & Z_{3 i}
\end{array}\right] \mathrm{I}_{2 i} \\
& \left.+\frac{\mu_{4}-\dot{\tau}_{2}(t)}{m} \sum_{i=1}^{m} \mathrm{I}_{2 i}^{T}\left[\begin{array}{cc}
X_{4 i} & Y_{4 i} \\
* & Z_{2 i}
\end{array}\right] \mathrm{I}_{2 i}\right\} \zeta(t) \\
& :=\zeta^{T}(t) \Lambda(t) \zeta(t),
\end{aligned}
$$

where $\Theta, \Xi, \Upsilon_{l}(l=1,2), \mathrm{I}_{k i}(k=1,2)$ are presented in (3.9), and

$$
\begin{aligned}
\zeta^{T}(t)=[ & x^{T}(t) \sigma_{1}^{T}\left(t-\tau_{1}\right) \sigma_{2}^{T}\left(t-\tau_{2}\right) x^{T}\left(t-\tau_{3}\right) \sigma^{T}\left(t-\tau_{1}-\rho_{1}(t)\right) \\
& \times \sigma^{T}\left(t-\tau_{2}-\rho_{2}(t)\right) f^{T}(x(t)) h_{1}^{T}\left(\sigma_{1}\left(t-\tau_{1}\right)\right) h_{2}^{T}\left(\sigma_{2}\left(t-\tau_{2}\right)\right) f^{T}\left(x\left(t-\tau_{3}\right)\right) \\
& \times h_{1}^{T}\left(\sigma_{1}\left(t-\tau_{1}-\rho_{1}(t)\right)\right) h_{2}^{T}\left(\sigma_{2}\left(t-\tau_{2}-\rho_{2}(t)\right)\right) \\
& \left.\times \dot{x}^{T}(t) x^{T}\left(t-\tau_{1}(t)\right) x^{T}\left(t-\tau_{2}(t)\right) f^{T}\left(x\left(t-\tau_{1}(t)\right)\right) f^{T}\left(x\left(t-\tau_{2}(t)\right)\right)\right] .
\end{aligned}
$$

Then utilizing the general convex technique in $[28,39]$, the LMIs described by (3.9) can guarantee $\Lambda(t)<0$, which indicates that there must exist a positive scalar $\chi>0$ such that

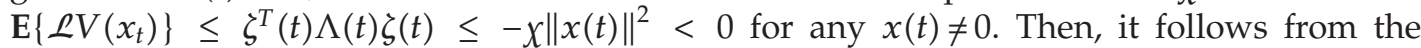
Lyapunov-Krasovskii stability theorem that the system (3.1) is asymptotically stable in the mean-square sense, and it completes the proof.

Remark 3.3. Transmitted delays are always existent in various dynamical networks due to the finite switching speed of amplifiers in electronic neural networks or the finite signal propagation time in biological networks. Furthermore, with the development of network delay tomography, the probability of time-delay distribution can be estimated. Thus, when the probability is available, it will be helpful to utilize such information to reduce the conservatism [29-35]. Yet, up till now, few authors have utilized the delay-partitioning idea to 
investigate the stability of DNNs with probabilistic time-varying delay, and, in this work, we applied one improved idea, which can fully consider the information of delay subinterval. Moreover, though the stability criterion in (3.9) is not presented in the forms of standard LMIs, it is still convenient and straightforward to check the feasibility without tuning any parameters by resorting to LMI in Matlab Toolbox.

Remark 3.4. Presently, in the literatures [21-28], various convex combination techniques have been widely employed and improved to tackle constant or time-varying delays owing to that they can help reduce the conservatism efficiently. In [41], the authors put forward the reciprocal convex approach, which can consider those important terms ignored and be more effective than the ones [21-28]. Yet it has come to our attention that the reciprocal convex one cannot efficiently tackle the case that both the bounds on delay derivatives are available. In this paper, we first combine the reciprocal convex technique and the convex ones to study the stability for DNNs with probabilistic time-varying delay.

Remark 3.5. As for $V_{2}\left(x_{t}\right)$ in (3.3), if we denote $\left[\begin{array}{cc}X_{2 i} & Y_{2 i} \\ * & Z_{2 i}\end{array}\right]=\left[\begin{array}{cc}X_{4 i} & Y_{4 i} \\ * & Z_{4 i}\end{array}\right]=0$ (resp., $\left[\begin{array}{ccc}X_{1 i} & Y_{1 i} \\ * & Z_{1 i}\end{array}\right]=$ $\left[\begin{array}{cc}X_{3 i} & Y_{3 i} \\ * & Z_{3 i}\end{array}\right]=0$ ), our results can be true as only $\mu_{2}, \mu_{4}$ (resp., $\left.\mu_{1}, \mu_{3}\right)$ are available. If we set $\left[\begin{array}{cc}X_{j i} & Y_{j i} \\ * & Z_{j i}\end{array}\right]=0(j=1,2,3,4)$ in (3.3) simultaneously, Theorem 3.2 still holds as that $\mu_{i}(i=$ $1,2,3,4)$ are unknown, or $\tau_{i}(t)(i=1,2)$ are not differentiable. Moreover, the number of freeweighting matrices in Theorem 3.2 is much smaller than the ones of these present results $[34,37]$, and the reciprocal convex technique is used, it can induce much more computational simplicity in a mathematical point of view.

Remark 3.6. In view of the delay-partitioning idea employed in this work, with integers $l, m$ increasing, the dimension of the derived LMIs will become higher and it will take more computing time to check them. Yet, if the lower bound of $\tau_{1}(t)$ is set and $l \geq 5$, the maximum allowable delay upper bound will become unapparently larger and approach to an approximate upper limitation [22-27]. Thus, if we want to employ the idea to real cases, we do not necessarily partition two delay intervals into too many segments.

Remark 3.7. In order to give the more general results, the delay intervals $\left[\tau_{1}, \tau_{2}\right]$ and $\left[\tau_{2}, \tau_{3}\right]$ are, respectively, divided into $l, m$ subintervals in this work, which makes the condition of Theorem 3.2 seem to be very complicated. Yet if we set $l=m \leq 5$, our results will avoid the complexity in some degree. Moreover, if we choose the simple Lyapunov-Krasovskii functional in (3.3) with $H_{1}=Y_{1 i}=Y_{2 i}=Y_{3 i}=Y_{4 i}=0$ and $\widetilde{H}_{i}=0$, the condition of Theorem 3.2 also will become much less complicated.

\section{Numerical Examples}

In this section, four numerical examples will be presented to illustrate the derived results. Firstly, we will utilize a numerical example to illustrate the significance of studying the lower bound of delay derivative.

Example 4.1. As a special case of $\delta_{0}=1$, we revisit the delayed neural networks considered in $[21,28]$ with

$$
C=\left[\begin{array}{ll}
2 & 0 \\
0 & 2
\end{array}\right], \quad A=\left[\begin{array}{cc}
1 & 1 \\
-1 & -1
\end{array}\right], \quad B=\left[\begin{array}{cc}
0.88 & 1 \\
1 & 1
\end{array}\right], \quad \Sigma=\left[\begin{array}{ll}
0 & 0 \\
0 & 0
\end{array}\right], \quad \bar{\Sigma}=\left[\begin{array}{cc}
0.4 & 0 \\
0 & 0.8
\end{array}\right],
$$


Table 1: Calculated MAUBs $\tau_{\max }$ for $l=1$, unavailable $\mu_{1}$ in Example 4.1.

\begin{tabular}{lcccc}
\hline Methods $\backslash \mu_{2}$ & 0.6 & 0.8 & 0.9 & 1.2 \\
\hline Theorem 3.2 [21] & 3.5209 & 2.8654 & 1.9508 & - \\
Theorem 3.2 [28] & 3.4877 & 2.8456 & 1.9149 & 1.1168 \\
Theorem 3.2 & 3.5102 & 2.8554 & 1.9447 & 1.1212 \\
\hline
\end{tabular}

Table 2: Calculated MAUBs $\tau_{\max }$ for $l=1$ and $\mu_{1}=0.5$ in Example 4.1.

\begin{tabular}{lcccr}
\hline Methods $\backslash \mu_{2}$ & 0.6 & 0.8 & 0.9 & 1.2 \\
\hline Theorem 3.2 [28] & 3.5871 & 2.8813 & 1.9652 & 1.2052 \\
Theorem 3.2 & 3.6198 & 2.9018 & 2.0012 & 1.2853 \\
\hline
\end{tabular}

and $\tau_{1}=0$ is set. If we do not consider the existence of $\mu_{1}$, then, by utilizing Theorem 3.2 and Remark 3.5, the corresponding maximum allowable upper bounds (MAUBs) $\tau_{\max }$ for different $\mu_{2}$ derived by the results in [21] and in the paper can be summarized in Table 1, which demonstrates that Theorem 3.2 of $l=1$ is somewhat more conservative than the one in $[21,28]$. Yet, if we set $\mu_{1}=0.5$, it is easy to verify that our results can yield much less conservative results than the ones in $[21,28]$, which can be shown in Table 2.

Based on Tables 1 and 2, it is indicated that the conservatism of stability criterion can be greatly deduced if we take the available $\mu_{1}$ into consideration. Moreover, though the delaypartitioning idea has been used in [28], the corresponding MAUBs $\tau_{\max }$ derived by [28] and Theorem 3.2 are summarized in Table 3, which shows that our idea can be more efficient than the one in [18] even for $l=1,2$.

Example 4.2. Considering the special case of $\delta_{0}=1$, we consider the delayed neural networks (2.1) with

$$
\begin{aligned}
C & =\operatorname{diag}\{1.2769,0.6231,0.9230,0.4480\}, \\
\Sigma & =0_{3 \times 3,} \bar{\Sigma}=\operatorname{diag}\{0.1137,0.1279,0.7994,0.2368\}, \\
A & =\left[\begin{array}{cccc}
-0.0373 & 0.4852 & -0.3351 & 0.2336 \\
-1.6033 & 0.5988 & -0.3224 & 1.2352 \\
0.3394 & -0.0860 & -0.3824 & -0.5785 \\
-0.1311 & 0.3253 & -0.9534 & -0.5015
\end{array}\right], \\
B & =\left[\begin{array}{cccc}
0.8674 & -1.2405 & -0.5325 & 0.0220 \\
0.0474 & -0.9164 & 0.0360 & 0.9816 \\
1.8495 & 2.6117 & -0.3788 & 0.8428 \\
-2.0413 & 0.5179 & 1.1734 & -0.2775
\end{array}\right]
\end{aligned}
$$

which has been addressed extensively; see [26, 27] and the references therein. Together with the delay-partitioning idea and for different $\mu_{2}$, the work [27] has calculated the MAUBs $\tau_{\max }$ such that the origin of the system is globally asymptotically stable for $\tau_{1}(t)$ satisfying $3=\tau_{1} \leq \tau_{1}(t) \leq \tau_{2} \leq \tau_{\max }$. By resorting to Theorem 3.2 and Remark 3.5, the corresponding results can be given Table 4 , which indicates that our delay-partitioning idea can be more effective than the relevant ones in [27] even for $l=1,2$ and $\mu_{1}=0$. 
Table 3: Calculated MAUBs $\tau_{\max }$ for various $l$ and $\mu_{1}=0.5$ in Example 4.1.

\begin{tabular}{lccc}
\hline Methods $\backslash \mu_{2}$ & 0.8 & 0.9 & unknown $\mu_{2}$ \\
\hline $\begin{array}{l}\text { Theorem 3.2 [28] } \\
l=1\end{array}$ & 2.8813 & & 1.2052 \\
$\quad l=2$ & 3.1486 & 1.9655 & 1.4073 \\
$\begin{array}{l}\text { Theorem 3.2 } \\
l=1\end{array}$ & 2.8873 & & \\
$l=2$ & 3.1678 & 1.9677 & 1.2100 \\
\hline
\end{tabular}

Table 4: Calculated MAUBs $\tau_{\max }$ for various $l, \mu_{2}$ in Example 4.2.

\begin{tabular}{|c|c|c|c|c|}
\hline Methods $\backslash \mu_{2}$ & 0.1 & 0.5 & 0.9 & unknown $\mu_{2}$ \\
\hline \multicolumn{5}{|c|}{ Theorem 3.2 [27] } \\
\hline$l=1$ & 3.33 & 3.16 & 3.10 & 3.09 \\
\hline$l=2$ & 3.65 & 3.32 & 3.26 & 3.24 \\
\hline \multicolumn{5}{|l|}{ Theorem 3.2} \\
\hline$l=1$ & 3.35 & 3.22 & 3.15 & 3.13 \\
\hline$l=2$ & 3.77 & 3.42 & 3.34 & 3.32 \\
\hline
\end{tabular}

Example 4.3. We still consider the DNNs with the following parameters [24, 28] by setting $\delta_{0}=1$ :

$$
\begin{array}{ll}
C & =\left[\begin{array}{ccc}
4.1889 & 0 & 0 \\
0 & 0.7160 & 0 \\
0 & 0 & 1.9985
\end{array}\right], \quad B=\left[\begin{array}{ccc}
-0.1052 & -0.5069 & -0.1121 \\
-0.0257 & -0.2808 & 0.0212 \\
0.1205 & -0.2153 & 0.1315
\end{array}\right], \\
\bar{\Sigma} & =\left[\begin{array}{ccc}
0.4129 & 0 & 0 \\
0 & 3.8993 & 0 \\
0 & 0 & 1.016
\end{array}\right],
\end{array}
$$

$A=\Sigma=0_{3 \times 3}$, and time-varying delay $\tau(t)$ satisfies $0 \leq \tau(t) \leq \tau_{2}, 0 \leq \dot{\tau}(t) \leq \mu_{2}$. Then, the MAUBs for this example with time-varying delay $\tau(t)$ are given in Table 5 by using the delay-partitioning idea in [28] and the one in this paper, which can illustrate that our delaydecomposition idea is superior over the one by [28].

Example 4.4. Consider DNN (2.3) of the following parameters, which has been considered in [33]:

$$
C=\left[\begin{array}{ll}
7 & 0 \\
0 & 6
\end{array}\right], \quad A=\left[\begin{array}{ll}
0.2 & -4 \\
0.1 & 0.3
\end{array}\right], \quad B=\left[\begin{array}{ll}
0.4 & 0.2 \\
0.1 & 0.7
\end{array}\right], \quad f(x)=\left[\begin{array}{c}
\tanh \left(-0.2 x_{1}\right) \\
\tanh \left(x_{2}\right)
\end{array}\right]
$$

If we set $\tau_{1}=0, \tau_{2}=0.4, \mu_{1}=\mu_{3}=0.1$, and $\mu_{2}=\mu_{4}$, the relevant MAUBs $\tau_{\max }$ are computed and listed in Tables 6 and 7 by setting $l=m=1$, for various $\mu_{2}$, and the delay probability distribution $\delta_{0}=0.2$, which can guarantee the addressed system to be asymptotically stable in the mean-squared sense. 
Table 5: Calculated MAUBs $\tau_{\max }$ for $l=2, \mu_{2}$ in Example 4.3.

\begin{tabular}{lcccr}
\hline Methods $\backslash \mu_{2}$ & 0.1 & 0.5 & 0.9 & 1 \\
\hline Theorem 3.2 [28] & 2.0011 & 1.3450 & 1.0687 & 1.0685 \\
Theorem 3.2 & 2.0148 & 1.3485 & 1.0701 & 1.0698 \\
\hline
\end{tabular}

Table 6: Calculated MAUBs $\tau_{\max }$ for $l=m=1$, various $\mu_{2}$, and $\delta_{0}=0.2$ in Example 4.4.

\begin{tabular}{lccccc}
\hline Methods $\backslash \mu_{2}$ & 0.2 & 0.6 & 1 & 1.5 & 2.5 \\
\hline Theorem 3.2 [33] & 1.1281 & 1.1279 & 1.1278 & 1.1277 & 1.1276 \\
Theorem 3.2 [28] & 1.3149 & 1.3086 & 1.3031 & 1.3026 & 1.3025 \\
Theorem 3.2 & 1.4242 & 1.4189 & 1.4134 & 1.4131 & 1.4130 \\
\hline
\end{tabular}

Table 7: Calculated MAUBs $\tau_{\max }$ for $l=m=1$, various $\mu_{2}$, and $\delta_{0}=0.8$ in Example 4.4.

\begin{tabular}{lccccc}
\hline Methods $\backslash \mu_{2}$ & 0.2 & 0.6 & 1 & 1.5 & 2.5 \\
\hline Theorem 3.2 [33] & 1.7177 & 1.6869 & 1.5758 & 1.5757 & 1.5757 \\
Theorem 3.2 [28] & 1.9778 & 1.8863 & 1.7742 & 1.7741 & 1.7740 \\
Theorem 3.2 & 2.0285 & 1.9678 & 1.9112 & 1.8785 & 1.7876 \\
\hline
\end{tabular}

Based on Tables 6 and 7, even for $m=l=1$, our results still can be less conservative than the one in [33]. As for $m=1, l=2$, one verifies that our results can reduce the conservatism much more evidently.

\section{Conclusions}

This paper has investigated the asymptotical stability for DNNs with probability-distribution delay. Through employing an improved idea of delay partitioning and constructing one novel Lyapunov-Krasovskii functional, one stability criterion with significantly reduced conservatism has been established in terms of LMIs. The proposed stability condition benefits from both bounds of time-varying delay and variation and combined convex technique. Four numerical examples have been given to demonstrate the effectiveness of the derived criteria and the improvements over some existent ones.

\section{Acknowledgments}

This work is supported by the National Natural Science Foundation of China no. 60904020, no. 61004064, and no. 61004032 and the Special Foundation of China Postdoctoral Science Foundation Project no. 201003546.

\section{References}

[1] Y. Zhao, L. Zhang, S. Shen, and H. Gao, "Robust stability criterion for discrete-time uncertain markovian jumping neural networks with defective statistics of modes transitions," IEEE Transactions on Neural Networks, vol. 22, no. 1, pp. 164-170, 2011.

[2] Z. Zuo, C. Yang, and Y. Wang, "A new method for stability analysis of recurrent neural networks with interval time-varying delay," IEEE Transactions on Neural Networks, vol. 21, no. 2, pp. 339-344, 2010.

[3] R. Rakkiyappan, P. Balasubramaniam, and J. Cao, "Global exponential stability results for neutraltype impulsive neural networks," Nonlinear Analysis, vol. 11, no. 1, pp. 122-130, 2010. 
[4] M. Syed Ali and P. Balasubramaniam, "Stability analysis of uncertain fuzzy Hopfield neural networks with time delays," Communications in Nonlinear Science and Numerical Simulation, vol. 14, no. 6, pp. 2776-2783, 2009.

[5] J. Tian, D. Xu, and J. Zu, "Novel delay-dependent asymptotic stability criteria for neural networks with time-varying delays," Journal of Computational and Applied Mathematics, vol. 228, no. 1, pp. 133$138,2009$.

[6] N. Ozcan and S. Arik, "A new sufficient condition for global robust stability of bidirectional associative memory neural networks with multiple time delays," Nonlinear Analysis, vol. 10, no. 5, pp. 3312-3320, 2009.

[7] P. Balasubramaniam, V. Vembarasan, and R. Rakkiyappan, "Delay-dependent robust exponential state estimation of Markovian jumping fuzzy Hopfield neural networks with mixed random timevarying delays," Communications in Nonlinear Science and Numerical Simulation, vol. 16, no. 4, pp. 2109 2129, 2011.

[8] P. Balasubramaniam, R. Krishnasamy, and R. Rakkiyappan, “Delay-dependent stability of neutral systems with time-varying delays using delay-decomposition approach," Applied Mathematical Modelling, vol. 36, no. 5, pp. 2253-2261, 2012.

[9] P. Balasubramaniam, V. Vembarasan, and R. Rakkiyappan, "Leakage delays in T-S fuzzy cellular neural networks," Neural Processing Letters, vol. 33, no. 2, pp. 111-136, 2011.

[10] H. Li, "Synchronization stability for discrete-time stochastic complex networks with probabilistic interval time-varying delays," International Journal of Innovative Computing, Information and Control, vol. 7, no. 2, pp. 697-708, 2011.

[11] H. Li, "Cluster synchronization stability for stochastic complex dynamical networks with probabilistic interval time-varying delays," Journal of Physics A, vol. 44, no. 10, p. 105101, 24, 2011.

[12] H. Li, W. K. Wong, and Y. Tang, "Global synchronization stability for stochastic complex dynamical networks with probabilistic interval time-varying delays," Journal of Optimization Theory and Applications, vol. 152, no. 2, pp. 496-516, 2012.

[13] Z. Zhang, W. Liu, and D. Zhou, "Global asymptotic stability to a generalized Cohen-Grossberg BAM neural networks of neutral type delays," Neural Networks, vol. 25, pp. 94-105, 2012.

[14] Z. Zhang and D. Zhou, "Global robust exponential stability for second-order Cohen-Grossberg neural networks with multiple delays," Neurocomputing, vol. 73, no. 1-3, pp. 213-218, 2009.

[15] Z. Zhang, Y. Yang, and Y. Huang, "Global exponential stability of interval general BAM neural networks with reaction-diffusion terms and multiple time-varying delays," Neural Networks, vol. 24, no. 5, pp. 457-465, 2011.

[16] Z. Zhang, K. Liu, and Y. Yang, “New LMI-based condition on global asymptotic stability concerning BAM neural networks of neutral type," Neurocomputing, vol. 81, pp. 24-32, 2012.

[17] X. Li and X. Fu, "Global asymptotic stability of stochastic Cohen-Grossberg-type BAM neural networks with mixed delays: an LMI approach," Journal of Computational and Applied Mathematics, vol. 235, no. 12, pp. 3385-3394, 2011.

[18] X. Li, "Global robust stability for stochastic interval neural networks with continuously distributed delays of neutral type," Applied Mathematics and Computation, vol. 215, no. 12, pp. 4370-4384, 2010.

[19] X. Li and R. Rakkiyappan, "Delay-dependent global asymptotic stability criteria for stochastic genetic regulatory networks with Markovian jumping parameters," Applied Mathematical Modelling, vol. 36, no. 4, pp. 1718-1730, 2012.

[20] X. Li, X. Fu, P. Balasubramaniam, and R. Rakkiyappan, "Existence, uniqueness and stability analysis of recurrent neural networks with time delay in the leakage term under impulsive perturbations," Nonlinear Analysis, vol. 11, no. 5, pp. 4092-4108, 2010.

[21] Y. Zhang, D. Yue, and E. Tian, "New stability criteria of neural networks with interval time-varying delay: a piecewise delay method," Applied Mathematics and Computation, vol. 208, no. 1, pp. 249-259, 2009.

[22] S. Mou, H. Gao, J. Lam, and W. Qiang, "A new criterion of delay-dependent asymptotic stability for Hopfield neural networks with time delay," IEEE Transactions on Neural Networks, vol. 19, no. 3, pp. 532-535, 2008.

[23] R. Yang, H. Gao, and P. Shi, "Novel robust stability criteria for stochastic Hopfield neural networks with time delays," IEEE Transactions on Systems, Man, and Cybernetics B, vol. 39, no. 2, pp. 467-474, 2009.

[24] H. Zhang, Z. Liu, G. B. Huang, and Z. Wang, "Novel weighting-delay-based stability criteria for recurrent neural networks with time-varying delay," IEEE Transactions on Neural Networks, vol. 21, no. 1, pp. 91-106, 2010. 
[25] W. H. Chen and W. X. Zheng, "Improved delay-dependent asymptotic stability criteria for delayed neural networks," IEEE Transactions on Neural Networks, vol. 19, no. 12, pp. 2154-2161, 2008.

[26] X. M. Zhang and Q. L. Han, "New Lyapunov-Krasovskii functionals for global asymptotic stability of delayed neural networks," IEEE Transactions on Neural Networks, vol. 20, no. 3, pp. 533-539, 2009.

[27] L. Hu, H. Gao, and W. X. Zheng, "Novel stability of cellular neural networks with interval timevarying delay," Neural Networks, vol. 21, no. 10, pp. 1458-1463, 2008.

[28] G. B. Zhang, T. Wang, T. Li, and S. M. Fei, "Delay-derivative-dependent stability criterion for neural networks with proba- bilistic time-varying delay," International Journal of Systems Science. In press.

[29] Y. Zhang, D. Yue, and E. Tian, "Robust delay-distribution-dependent stability of discrete-time stochastic neural networks with time-varying delay," Neurocomputing, vol. 72, no. 4-6, pp. 1265-1273, 2009.

[30] D. Yue, Y. Zhang, E. Tian, and C. Peng, "Delay-distribution-dependent exponential stability criteria for discrete-time recurrent neural networks with stochastic delay," IEEE Transactions on Neural Networks, vol. 19, no. 7, pp. 1299-1306, 2008.

[31] Y. Tang, J. A. Fang, M. Xia, and D. Yu, “Delay-distribution-dependent stability of stochastic discretetime neural networks with randomly mixed time-varying delays," Neurocomputing, vol. 72, no. 16-18, pp. 3830-3838, 2009.

[32] Y. Zhao, H. Gao, J. Lam, and K. Chen, "Stability analysis of discrete-time recurrent neural networks with stochastic delay," IEEE Transactions on Neural Networks, vol. 20, no. 8, pp. 1330-1339, 2009.

[33] J. Fu, H. Zhang, and T. Ma, "Delay-probability-distribution-dependent robust stability analysis for stochastic neural networks with time-varying delay," Progress in Natural Science, vol. 19, no. 10, pp. 1333-1340, 2009.

[34] M. S. Mahmoud, S. Z. Selim, and P. Shi, “Global exponential stability criteria for neural networks with probabilistic delays," IET Control Theory \& Applications, vol. 4, no. 11, pp. 2405-2415, 2010.

[35] H. Bao and J. Cao, "Delay-distribution-dependent state estimation for discrete-time stochastic neural networks with random delay," Neural Networks, vol. 24, no. 1, pp. 19-28, 2011.

[36] C. Peng, D. Yue, E. Tian, and Z. Gu, "A delay distribution based stability analysis and synthesis approach for networked control systems," Journal of the Franklin Institute. Engineering and Applied Mathematics, vol. 346, no. 4, pp. 349-365, 2009.

[37] D. Yue, E. Tian, Z. Wang, and J. Lam, "Stabilization of systems with probabilistic interval input delays and its applications to networked control systems," IEEE Transactions on Systems, Man, and Cybernetics Part A:Systems and Humans, vol. 39, no. 4, pp. 939-945, 2009.

[38] D. Yue, E. Tian, Y. Zhang, and C. Peng, "Delay-distribution-dependent robust stability of uncertain systems with time-varying delay," International Journal of Robust and Nonlinear Control, vol. 19, no. 4, pp. 377-393, 2009.

[39] D. Yue, E. Tian, Y. Zhang, and C. Peng, "Delay-distribution-dependent stability and stabilization of TS fuzzy systems with probabilistic interval delay," IEEE Transactions on Systems, Man, and Cybernetics B, vol. 39, no. 2, pp. 503-516, 2009.

[40] E. Fridman, U. Shaked, and K. Liu, "New conditions for delay-derivative-dependent stability," Automatica, vol. 45, no. 11, pp. 2723-2727, 2009.

[41] P. Park, J. W. Ko, and C. Jeong, "Reciprocally convex approach to stability of systems with timevarying delays," Automatica, vol. 47, no. 1, pp. 235-238, 2011.

[42] S. Cong and Y. Zou, "A new delay-dependent exponential stability criterion for Itô stochastic systems with Markovian switching and time-varying delay," International Journal of Systems Science. Principles and Applications of Systems and Integration, vol. 41, no. 12, pp. 1493-1500, 2010. 


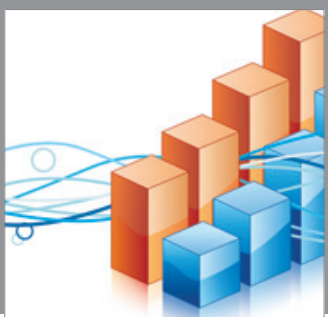

Advances in

Operations Research

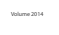

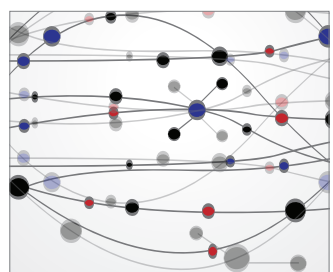

\section{The Scientific} World Journal
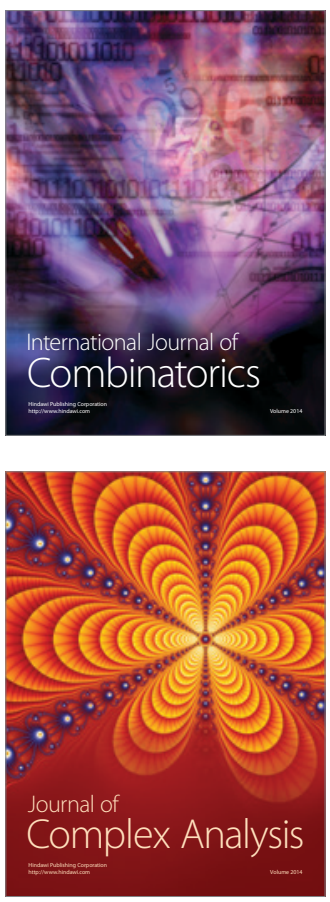

International Journal of

Mathematics and

Mathematical

Sciences
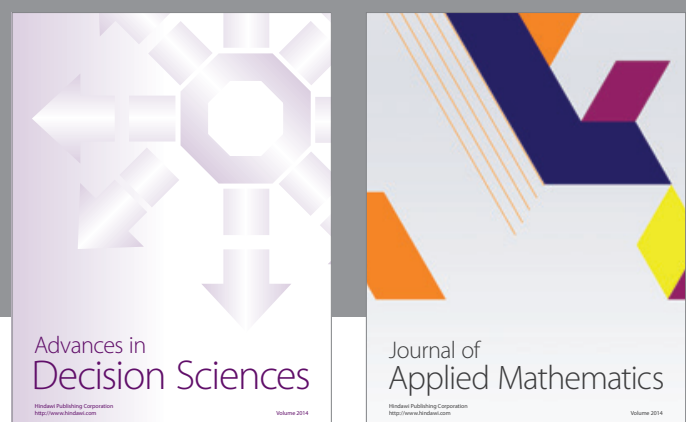

Journal of

Applied Mathematics
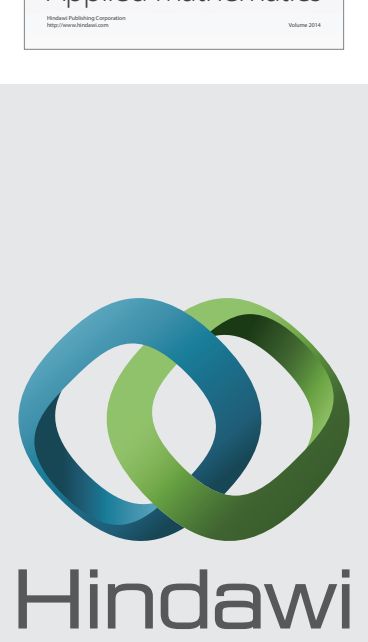

Submit your manuscripts at http://www.hindawi.com
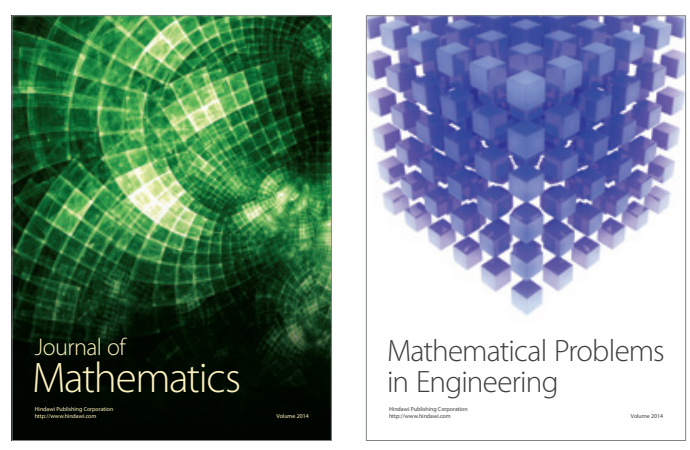

Mathematical Problems in Engineering
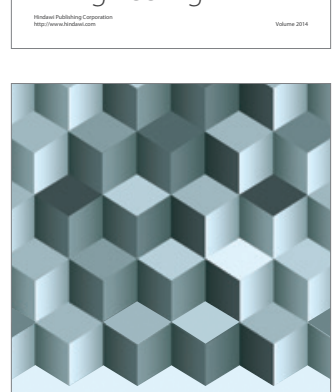

Journal of

Function Spaces
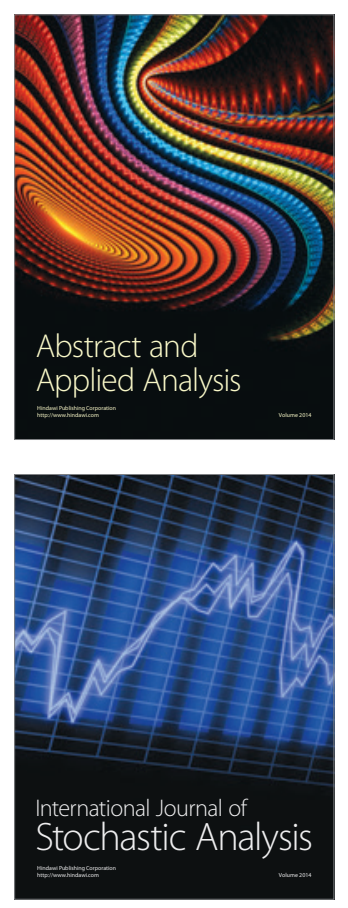

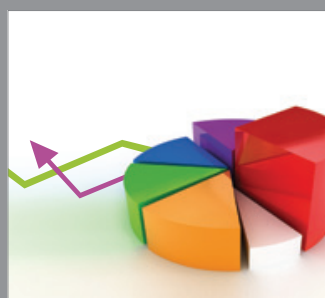

ournal of

Probability and Statistics

Promensencen
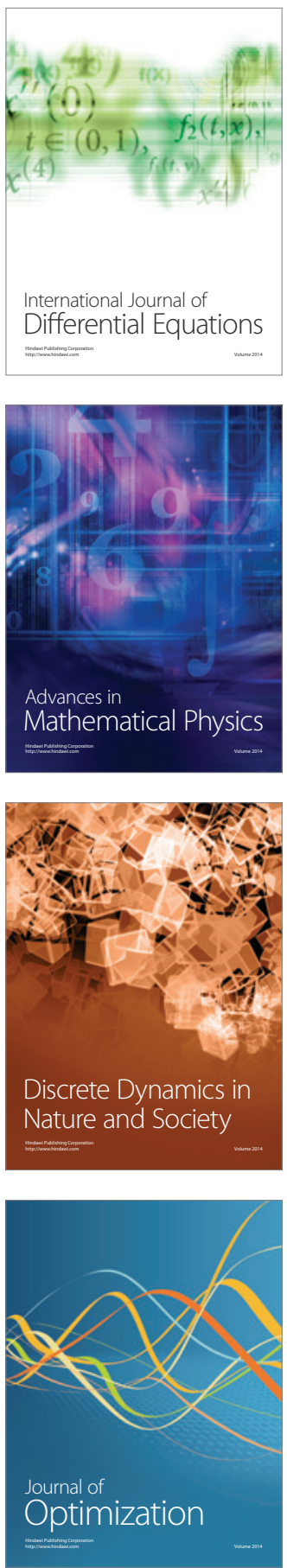